\title{
High Response Rate to Second-Line Combination Antiangiogenic Chemotherapy in Patients with Metastatic Melanoma
}

\author{
Ozan Haase ${ }^{1 * \#, ~ O z a n ~ A n g u ̈ n ~}{ }^{1 \#, ~ E w a n ~ A . ~ L a n g a n ~}{ }^{1,2}$, Franziska Hübner ${ }^{1}$, Florian M. Vogt ${ }^{3}$, \\ Christoph Thorns ${ }^{4}$, Detlef Zillikens ${ }^{1}$, Patrick Terheyden ${ }^{1}$ \\ ${ }^{1}$ Department of Dermatology, University of Lübeck, Lübeck, Germany \\ ${ }^{2}$ Comprehensive Centre for Inflammation Medicine, University of Lübeck, Lübeck, Germany \\ ${ }^{3}$ Department of Radiology, University of Lübeck, Lübeck, Germany \\ ${ }^{4}$ Institute of Pathology, Section for Hematopathology and Endocrine Pathology, University of Lübeck, Lübeck, Germany \\ Email: *ozan.haase@uksh.de
}

How to cite this paper: Haase, O., Angün, O., Langan, E.A., Hübner, F., Vogt, F.M., Thorns, C., Zillikens, D. and Terheyden, P. (2016) High Response Rate to Second-Line Combination Antiangiogenic Chemotherapy in Patients with Metastatic Melanoma. Journal of Cancer Therapy, 7, 908-918. http://dx.doi.org/10.4236/jct.2016.712088

Received: September 27, 2016

Accepted: November 8, 2016

Published: November 11, 2016

Copyright $\odot 2016$ by authors and Scientific Research Publishing Inc. This work is licensed under the Creative Commons Attribution International License (CC BY 4.0). http://creativecommons.org/licenses/by/4.0/ (c) (i) Open Access

\begin{abstract}
Objectives: Despite the array of new treatment strategies for the management of metastatic melanoma, the prognosis remains poor when immune checkpoint inhibition and targeted therapy options are exhausted. The antiangiogenic monoclonal antibody bevacizumab has documented its efficacy in the treatment of several solid tumors, when used in combination with standard chemotherapy. Phase II studies and case series have led to the speculation that it may also improve the prognosis when used in stage IV melanoma. Therefore, we investigated the influence of bevacizumab, combined with a platinum-based chemotherapy, in the treatment of melanoma and sought to identify prognostic factors affecting the response. Methods: Eight patients with metastatic melanoma, with documented progress after at least one previous therapy, received bevacizumab (5 $\mathrm{mg} / \mathrm{kg}$ intravenously every two weeks) in combination with cisplatin $\left(100 \mathrm{mg} / \mathrm{m}^{2}\right.$ every four weeks) and carboplatin $\left(200 \mathrm{mg} / \mathrm{m}^{2}\right.$ every four weeks). The therapy was continued until renewed disease progression occurred. The response rate, progression-free and overall survival, and toxicity were evaluated. Results: We observed complete remission in two patients (25\%) and partial response in an additional four patients. In one patient, the disease remained stable (total disease control rate of $87.5 \%)$. Only one patient (12.5\%) had progressive disease. The median progression-free survival was 6 months (range 3 - 37 months). The median overall survival time was 15.5 months (range 6 - 77 months). Every patient experienced at least one adverse event of grade 3 - 4, most commonly bleeding associated with severe thrombocytopenia. Conclusion: Our observations indicate that bevacizumab, in combination with cisplatin and carboplatin, may represent an effective treatment option for patients with metastatic melanoma and disease progression.
\end{abstract}

\#These authors have contributed equally to this work. 


\section{Keywords}

Melanoma, Cisplatin, Carboplatin, Bevacizumab, Response, Angiogenesis

\section{Introduction}

The recent development of agents which selectively target mutated genes or modulate immune responses has led to major advances in the therapy of metastatic melanoma [1]. However, there remains a sizable patient cohort, which develops progressive disease despite targeted therapy and immune check-point inhibition. Dacarbazine or polychemotherapy are therefore reserved for such patients with progressive disease, in whom targeted therapy and immune check-point inhibition options have been exhausted. Indeed, there are several studies which have examined the efficacy of platinum analogues as a part of combination chemotherapy in metastatic melanoma [2]. Güven et al. observed a response rate of $26.4 \%$ with a median survival time of 12.5 months using polychemotherapy with cisplatin and carboplatin in dacarbazine-resistant patients [3]. Hofmann et al. report a response rate of $14.2 \%$ with an overall survival of 32 weeks for partially heavily pretreated patients [4]. Such a polychemotherapy regime enables the total dose of platinum to be increased but the severe side effects of both single agents are avoided [3]. However, due to the limitations of the chemotherapeutic substances, there is still an urgent need for innovative and effective treatments. To this end, angiogenesis remains a viable therapeutic target [5].

Melanoma progression is dependent upon the induction of angiogenesis; vascular endothelial growth factor A (VEGF-A) is expressed in more than $80 \%$ of melanomas [6]. VEGF stimulates cell proliferation, survival and vascular maturation; by disrupting the vascular barrier function it leads also to tumour cell extravasation and causes vascular permeability and oedema. The abnormal properties of tumour-vessels can compromise drug delivery to the tumour. Hence it has been postulated that VEGF- inhibition improves the delivery of cytotoxic agents [7]. Bevacizumab, a humanized monoclonal antibody directed against VEGF-A, improved response rate and survival duration in various tumors in combination with chemotherapy [8]. At present, relatively little is known about the efficacy of bevacizumab, especially in combination with polychemotherapy, in patients with metastatic melanoma. However, the currently available published data suggest that addition of bevacizumab to platinum-based chemotherapy could be beneficial [7]. In fact, whilst a large randomized phase II clinical trial, comparing the combination of bevacizumab, paclitaxel and carboplatin to paclitaxel and carboplatin alone, failed to meet the primary objective of improved progression-free and overall survival, there was a clear trend towards a superior outcome for the triple combination [9]. An improvement was seen in almost all efficacy parameters, and even patients with increased lactate dehydrogenase (LDH) and M1c disease seemed to benefit from the additional antiangiogenic therapy. In light of these promising results, we performed treatment with bevacizumab in combination with cis- 
and carboplatin in eight patients with progressive disease to prior therapy.

\section{Methods}

The local ethics committee (University of Lübeck) was informed of the planned treatment intervention. Written informed consent was obtained prior to any treatment procedure. All patients had histologically confirmed and radiographically bi-dimensionally measurable unresectable metastatic melanoma as defined by the Response Evaluation Criteria in Solid Tumors (RECIST). Furthermore, all patients met the criteria of a performance status of $0-2$ as defined by the Eastern Cooperative Oncology Group (ECOG). Patients with partially extensively elevated liver enzymes or LDH values were also included. Prior to treatment, an audiogram was performed and renal impairment was excluded by the determination of the creatinine clearance. The BRAF mutational status was determined by Sanger sequencing in all patients. The primary tumor was used for analysis if possible. If not, the mutational status was determined in metastases. Patients with brain metastases were excluded. All patients were pretreated with standard chemotherapies (Table 1). Adverse events were evaluated according to the Common Terminology Criteria for Adverse Events v4.0 (CTCAE).

\subsection{Treatment Schedule}

The patients received $5 \mathrm{mg} / \mathrm{kg}$ bevacizumab (i.v.) fortnightly in combination with 100

Table 1. Baseline characteristics of the patients treated.

\begin{tabular}{cc}
\hline & Patients \\
Total & 8 \\
Sex, males/females & $3 / 5$ \\
Median age in years (range) & $57.5(26-77)$ \\
M-Classification & \\
M1a & $2(25.0 \%)$ \\
M1b & $1(12.5 \%)$ \\
M1c & $5(62.5 \%)$ \\
Elevated lactate dehydrogenase values & $4(50 \%)$ \\
BRAF V600E mutation & $1(12.5 \%)$ \\
Median number of prior therapies (range) & $1(1-2)$ \\
Kind of prior therapies & \\
DTIC & 7 \\
Paclitaxel & 1 \\
Immunotherapy (Allovectin-7) & 1 \\
Treosulfan/gemcitabine & 1 \\
Median number of applied cycles of & $5(3-17)$ \\
bevacizumab/cisplatin/carboplatin (range)
\end{tabular}


$\mathrm{mg} / \mathrm{m}^{2}$ cisplatin over 2 hours on day 1 and $200 \mathrm{mg} / \mathrm{m}^{2}$ carboplatin over 1 hour on day 2 every four weeks. Bevacizumab was administered on an outpatient basis, whereas chemotherapy was administered during an in-patient stay. The chemotherapy was administered as described previously [4]. Pre- and post-chemotherapy hydration was performed, before and after chemotherapy, with $1000 \mathrm{ml}$ normal saline (pre) and $1000 \mathrm{ml}$ 5\%-glucose solution (post) on both days. In the post-hydration phase, $40 \mathrm{mg}$ of furosemide was co-administered. The patients received granisetrone $3 \mathrm{mg}$, ranitidine $50 \mathrm{mg}$ and dexamethasone $20 \mathrm{mg}$ intravenously on both days prior to chemotherapy and granisetrone $2 \mathrm{mg}$ orally for the following 5 days. Patients with extensive nausea were additionally treated with aprepitant $125 \mathrm{mg}$ on the day before chemotherapy and $80 \mathrm{mg}$ on days $1-2$. The treatment was interrupted in case of grade 3 or 4 toxicity until toxicity grades reached 1 or 2 and the dosage of cisplatin and carboplatin was reduced by $25 \%$.

\subsection{Response Assessments}

The tumor status was assessed by CT scans of the chest and the abdomen every 12 weeks or earlier if clinically indicated. CT brain imaging was only performed when clinical suspicion of intracranial metastases arose. Response was evaluated using Response Evaluation Criteria in Solid Tumors (RECIST), version 1.1.

\section{Results}

\subsection{Patient Characteristics}

A total of eight patients were treated with bevacizumab combined with cis- and carboplatin. The characteristics of these patients are listed in Table 1. Patients had previously received at least one systemic treatment for metastatic disease, including vaccination- and chemotherapy. The median age at the beginning of the therapy was 57.5 years (range 26 - 77). Five patients were diagnosed with stage M1c, one with stage M1b and two with stage M1a melanoma. The median number of cycles delivered was 5 (range 3 - 17). A BRAF V600E mutation was detected in one patient.

\subsection{Adverse Events}

Dose reductions were required for all patients. The most common reason for dose reduction was grade 3 - 4 hematological toxicity. Five patients required blood transfusions, one patient needed a platelet transfusion. Epistaxis (grade 2) was observed in three patients. One patient experienced severe bleeding (grade 4) into a muscle metastasis. This metastasis was diagnosed on the upper left leg and had a diameter of $24 \mathrm{~cm}$. A complete resection of the metastasis was not possible as it encroached on the femoral artery. Two months later, leg amputation was necessary following growth of the metastasis (diameter of $10 \mathrm{~cm}$ ) and extensive bleeding into the tumor from the femoral artery.

Emesis was another common adverse event that was seen in seven patients. Hypertension occurred in three patients, three patients suffered from severe bacterial infections, including pneumonia $(n=2)$ and urosepsis $(n=1)$. Detailed information about the adverse events can be found in Table 2. 
Table 2. Adverse events.

\begin{tabular}{|c|c|c|c|c|c|}
\hline Adverse event & Grade 0 & Grade 1 & Grade 2 & Grade 3 & Grade 4 \\
\hline \multicolumn{6}{|c|}{ Blood } \\
\hline Anaemia & 0 & $1(12.5 \%)$ & $3(37.5 \%)$ & $4(50 \%)$ & 0 \\
\hline Leucocytopenia & $1(12.5 \%)$ & 0 & $2(25 \%)$ & $4(50 \%)$ & $1(12.5 \%)$ \\
\hline Thrombocytopenia & $1(12.5 \%)$ & $1(12.5 \%)$ & 0 & $2(25 \%)$ & $4(50 \%)$ \\
\hline \multicolumn{6}{|c|}{ Nervoussystem } \\
\hline Polyneuropathy & 0 & $2(25 \%)$ & $1(12.5 \%)$ & 0 & 0 \\
\hline \multicolumn{6}{|c|}{ Further adverse events } \\
\hline Hypertension & 0 & 0 & 0 & $3(37.5 \%)$ & 0 \\
\hline Allergy & 0 & 0 & 0 & $1(12.5 \%)$ & 0 \\
\hline Emesis & 0 & 0 & $4(50 \%)$ & $4(50 \%)$ & 0 \\
\hline Acute renal failure & 0 & 0 & 0 & $1(12.5 \%)$ & 0 \\
\hline Bleeding (tissue) & 0 & 0 & 0 & 0 & $1(12.5 \%)$ \\
\hline Bleeding (Epistaxis) & 0 & $3(37.5 \%)$ & 0 & 0 & 0 \\
\hline \multicolumn{6}{|l|}{ Infection (Pneumonia, } \\
\hline $\begin{array}{c}\text { Urosepsis, } \\
\text { Fungalsepsis) }\end{array}$ & 0 & 0 & 0 & $3(37.5 \%)$ & 0 \\
\hline
\end{tabular}

\subsection{Response Analysis}

We observed a response rate of $75 \%$. A complete response (CR) was observed in two patients (25\%) (Figure 1). Both of them had a single lymph node metastasis with normal LDH values and were pretreated with dacarbazine. A partial remission was documented in four patients (50\%) of whom three had elevated LDH values. One patient $(12.5 \%)$ had a stable disease and only one patient $(12.5 \%)$ had primary progressive disease. In summary, a disease control rate of $87.5 \%$ was observed. The median progression-free survival for all patients was 6 months (range 3 - 37). The median overall survival time was 15.5 months (range 3 - 77 months). In patients with stage M1c, the median survival time was 10 months compared to 21 months in stage M1a-M1b. The median survival in patients with elevated $\mathrm{LDH}$ values was 9 months compared to 20.5 months in patients with normal LDH values.

In one of the complete responders, chemotherapy was stopped after 9 cycles due to neurotoxicity in the absence of active disease. However, the melanoma relapsed 28 months later and chemotherapy with cisplatin, carboplatin and bevacizumab was reinitiated. 

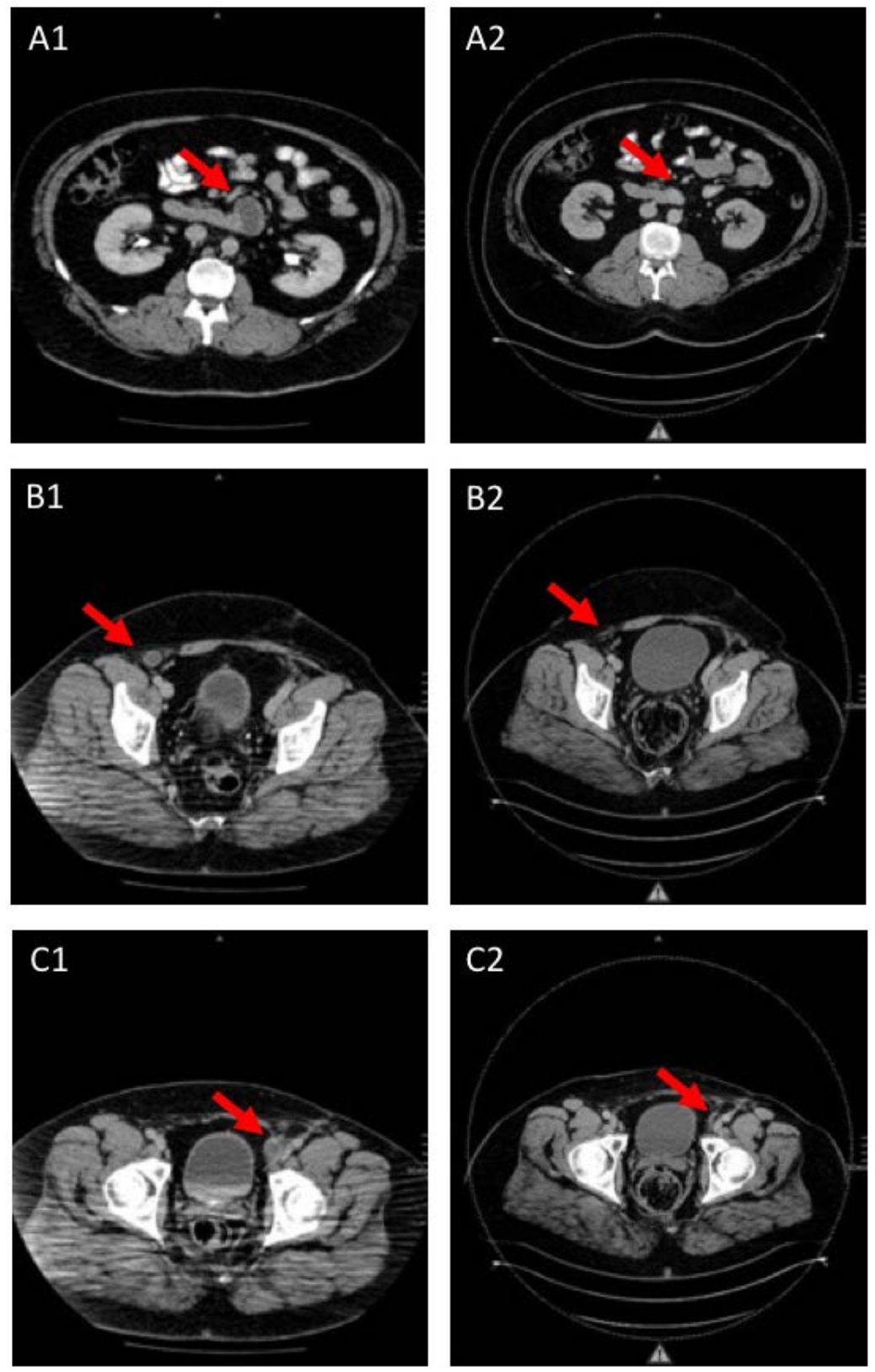

Figure 1. CT scans demonstrating complete response of lymph node metastasis after 3 courses in a 47-year-old patient (A1-C2). At the beginning of the treatment, there had been mesenteric (A1), inguinal (B1) and pelvic (C1) lymph node metastases with normal $\mathrm{LDH}$ and $\mathrm{S} 100$ values. The total number of courses administered was 5 . The treatment was stopped due to severe emesis. A2, B2 and C2 show the response that lasted over 5 months.

After six courses, the patient had a stable disease, surviving 77 months after the first dose of bevacizumab. The second patient with CR had a relapse only 3 months after the end of the therapy.

Only one patient demonstrated complete lack of response. This patient had a very high tumor burden with tumor manifestations in several sites including bone and liver. 
The patient with BRAF V600E mutated melanoma had a PFS and OS of 4 and 6 months, respectively, but still, this patient benefitted from treatment with a partial response.

\section{Discussion}

With the rise of the targeted therapies and checkpoint inhibition the management of melanoma has changed dramatically. Chemotherapy is no longer regarded as a reasonable first, or in the case of BRAF mutated melanoma, second-line therapy in melanoma patients [10] [11]. Nevertheless, chemotherapy remains an option after the new compounds lose their effect and the disease progresses. Even platinum-based polychemotherapy may be a favorable option.

Platinum analogues have played an important role in the treatment of dacarbazine-resistant melanoma [3]. The combination of chemotherapy with the VEGF-inhibitor bevacizumab is based on the highly vascularized nature of melanoma and the roles VEGF plays both in the angiogenetic stimulation of melanoma progression as well as in the resistance to cytotoxic drugs [12]. Moreover, inhibition of VEGF may play a role in the combination with immune checkpoint inhibitors by promoting the infiltration of activated $\mathrm{T}$ cells into tumors [13] [14].

Compared to the outcome parameters for pretreated patients that have been published for the combination of cis- and carboplatin alone [3], our small cohort of stage IV melanoma patients treated with cisplatin, carboplatin and bevacizumab demonstrated promising results in terms of ORR and OS. Indeed, we observed a $25 \%$ CR rate, a $50 \%$ PR rate, a disease control rate of $87.5 \%$, and a median OS of 15.5 months. In the cohort of Güven et al., lacking bevacizumab therapy, the CR and PR rate was 13.3\% respectively, the disease control rate was $52.8 \%$, and the OS was 12.5 months. However, the median PFS was shorter in our population (6 months vs. 7.1 months). The improved ORR and OS are remarkable considering a M1c rate of $62.5 \%$ and elevated $\mathrm{LDH}$ rate of $50 \%$ in our cohort, generally accepted as poor prognostic factors for treatment response in chemotherapy trials. In the cohort of Güven et al. the M1c rate was comparable with $73.3 \%$ (the elevated $\mathrm{LDH}$ rate was not given in the report). Even though a response in patients with high $\mathrm{LDH}$ values was shown here, there is a tendency towards patients with markedly elevated LDH levels experiencing a poorer outcome as previously described [15].

In our cohort we could identify only one patient $(12.5 \%)$ with an activating BRAF V600E mutation, far more infrequent than described in other studies [16] [17]. This patient achieved a PFS survival and OS of 4 and 6 months, but still had a benefit from treatment with a PR. Interestingly, BRAF mutated patients seem to achieve poorer outcomes under the chemotherapy plus bevacizumab combination [18].

Real-world outcome results of various chemotherapy options, in the pre-ipilimimab era, were reported with a median PFS of 2.6 months, a median OS of 8.8 months and an OR rate of only 7\% [19]. All of these parameters were exceeded by our results as well as other bevacizumab and chemotherapy combinations. Response rates of up to $36 \%$ of melanoma patients treated with bevacizumab in combination with paclitaxel were ob- 
served in small cohorts by other authors (Table 3). These studies were phase II trials in the first-line situation demonstrating highest response rates when a platinum analogue was combined. The BEAM trial, the largest study to date in which bevacizumab was tested in metastatic melanoma, evaluated patients receiving bevacizumab in combination with carboplatin and paclitaxel compared to carboplatin, paclitaxel and placebo as a first line treatment [9]. The PFS was 5.6 months in the bevacizumab arm compared to 4.2 months in the control group. The OS was significantly increased in the bevacizumab group with 12.3 months compared to 8.6 months. With $25.5 \%$ the response rate was also significantly higher than in the control group with $16.4 \%$. Despite clear trends for effectiveness the study was considered to be unsuccessful, because the increase of PFS was chosen as the primary end point. One may speculate that a phase III trial would provide new insights into this issue [20]. Furthermore, the BEAM trial demonstrated a favorable outcome for both PFS and OS for the bevacizumab-treated patients with elevated $\mathrm{LDH}$ values, the group most treatment resistant. This observation was confirmed by the Swiss phase 2 study that investigated the first-line combination of bevacizumab with temozolomide (Table 3) [18]. In our cohort, three patients with elevated LDH (75\%) achieved PR and a median OS of 9 months. Perhaps patients with more aggressive tumor growth and an immunosuppressive tumor microenvironment may profit the most from bevacizumab combinations. This could be explained by the hypoxia- reducing effect and improved drug and immune cell delivery [13] [21] [22]. Interestingly, the combination of bevacizumab with ipilimumab demonstrated an impressive median PFS of 9 months and a response rate of $20 \%$ in 46 melanoma patients [14]. On the other hand, consistent with the published literature, our data demonstrate the most favorable outcome in $\mathrm{Mla} / \mathrm{b}$ and low serum LDH concentrations patients.

While bevacizumab shows promising results in combination therapy, it may not be sufficient for monotherapy. The report of the interim results of the large adjuvant bevacizumab monotherapy trial (1343 patients) described only marginal trends for the bevacizumab-treated patients in comparison to untreated patients [23].

The combination of cispatin, carboplatin and bevacizumab is accompanied by a high rate of adverse events. The more courses are administered, the more likely haematotox-

Table 3. Melanoma therapy trials with bevacizumab.

\begin{tabular}{ccccccc}
\hline Trial & First/Second & Line & RR (\%) & PFS (mo) & OS (mo) & Ref. \\
Bevacizumab + paclitaxel & Second & 12 & 16.6 & 3.7 & n.g. & {$[25]$} \\
Bevacizumab + carboplatin + paclitaxel & First & 53 & 17 & 6 & 12 & {$[26]$} \\
\hline Bevacizumab + carboplatin + paclitacel vs. placebo + carboplatin + paclitaxel & First & 214 & $25.5 / 16.4$ & $5.6 / 4.2$ & $12.3 / 8.6$ & {$[9]$} \\
Bevacizumab + temozolomide vs bevacizumab + nab-paclitaxel + carboplatin & First & $42 / 51$ & $23.8 / 33.3$ & $3.8 / 6.7$ & $12.3 / 13.9$ & {$[27]$} \\
Bevacizumab + temozolomide & First & 62 & 16.1 & 4.2 & 9.6 & {$[18]$} \\
Bevacizumab + nab-paclitaxel & First & 50 & 36 & 7.6 & 16.8 & {$[28]$} \\
Bevacizumab + ipilimumab & First & 46 & 20 & 9 & 25.1 & {$[14]$} \\
Bevacizumab + dacarbazine & First & 37 & 19 & 5.5 & 11.4 & {$[29]$} \\
\hline
\end{tabular}


icity and irreversible neurotoxicity will develop. Bevacizumab-associated thrombocytopenia and the subsequent bleeding risk are of special concern. The most common adverse event in our patient group was reversible grade 3 - 4 haematotoxicity which led to dose reduction (Table 2). Cisplatin is known for its nephrotoxic potential but in our case series, we only observed mild grade I-II nephrotoxic side effects which may be due to adequate pre- and post-hydration. The most feared adverse event associated with bevacizumab is severe bleeding especially of the gastrointestinal tract. We observed mild to severe bleeding in four of our patients (50\%). Symptoms ranged from epistaxis to severe bleeding of a tumor. In three of our patients we observed hypertension, most likely induced by bevacizumab. It is possible that hypertension accompanying the use of antiangiogenic substances could be a favorable predictive factor [24]. All 3 patients that experienced hypertension had also a partial remission in our case series, but yet, PFS and OS was not improved when compared to the responders without hypertension. The hypertension was treated with antihypertensives and the patients continued bevacizumab application. The BEAM trial showed a significantly reduced risk of neutropenia, peripheral neuropathy and hypertension when bevacizumab was added to chemotherapy, although these adverse advents occurred less often than in our patient group treated in combination with cisplatin and carboplatin [9].

\section{Conclusion}

The combined chemotherapy with bevacizumab, cisplatin and carboplatin showed beneficial effects in the treatment of stage IV melanoma. We assessed pretreated patients with a poor prognosis and observed a very high response rate that could be of value for symptomatic and rapidly progressive disease. However, the risk of severe thrombopenia and bleeding must be considered. Although encouraging results regarding the response to this therapy are reported, our observation is limited by the small sample size. Future controlled studies may assess whether and to what extent combined chemotherapy with bevacizumab, cisplatin and carboplatin may be an option for patients who do not respond to checkpoint inhibition or develop resistance to targeted therapy.

\section{Conflicts of Interest and Source of Funding}

P. T. received honoraria from BMS, GSK, Novartis, MSD and Roche. For all other authors none were declared.

\section{References}

[1] Dummer, R., Schadendorf, D., Ascierto, P.A., Larkin, J., Lebbe, C. and Hauschild, A. (2015) Integrating First-Line Treatment Options into Clinical Practice: What's New in Advanced Melanoma? Melanoma Research, 25, 461-469. http://dx.doi.org/10.1097/CMR.0000000000000200

[2] Garbe, C., Eigentler, T.K., Keilholz, U., Hauschild, A. and Kirkwood, J.M. (2011) Systematic Review of Medical Treatment in Melanoma: Current Status and Future Prospects. The Oncologist, 16, 5-24. http://dx.doi.org/10.1634/theoncologist.2010-0190 
[3] Guven, K., Kittler, H., Wolff, K. and Pehamberger, H. (2001) Cisplatin and Carboplatin Combination as Second-Line Chemotherapy in Dacarbazine-Resistant Melanoma Patients. Melanoma Research, 11, 411-415. http://dx.doi.org/10.1097/00008390-200108000-00012

[4] Hofmann, M.A., Gabriel, V., Milling, A., Kiecker, F., Sterry, W. and Trefzer, U. (2007) High-Dose Platinum Combination Therapy in Pretreated Patients with Disseminated Melanoma. Chemotherapy, 53, 422-428. http://dx.doi.org/10.1159/000110007

[5] Garber, K. (2014) Promising Early Results for Immunotherapy-Antiangiogenesis Combination. Journal of the National Cancer Institute, 106, Article ID: dju392. http://dx.doi.org/10.1093/jnci/dju392

[6] Tu, Y.T., Tao, J., Liu, Y.Q., et al. (2006) Expression of Endothelial Nitric Oxide Synthase and Vascular Endothelial Growth Factor in Human Malignant Melanoma and Their Relation to Angiogenesis. Clinical and Experimental Dermatology, 31, 413-418. http://dx.doi.org/10.1111/j.1365-2230.2006.02123.x

[7] Terheyden, P., Hofmann, M.A., Weininger, M., Brocker, E.B. and Becker, J.C. (2007) AntiVascular Endothelial Growth Factor Antibody Bevacizumab in Conjunction with Chemotherapy in Metastasising Melanoma. Journal of Cancer Research and Clinical Oncology, 133, 897-901. http://dx.doi.org/10.1007/s00432-007-0251-8

[8] Jayson, G.C., Kerbel, R., Ellis, L.M. and Harris, A.L. (2016) Antiangiogenic Therapy in Oncology: Current Status and Future Directions. The Lancet, 388, 518-529. http://dx.doi.org/10.1016/S0140-6736(15)01088-0

[9] Kim, K.B., Sosman, J.A., Fruehauf, J.P., et al. (2012) BEAM: A Randomized Phase II Study Evaluating the Activity of Bevacizumab in Combination with Carboplatin plus Paclitaxel in Patients with Previously Untreated Advanced Melanoma. Journal of Clinical Oncology, 30, 34-41. http://dx.doi.org/10.1200/JCO.2011.34.6270

[10] Ribas, A., Puzanov, I., Dummer, R., et al. (2015) Pembrolizumab versus Investigator-Choice Chemotherapy for Ipilimumab-Refractory Melanoma (KEYNOTE-002): A Randomised, Controlled, Phase 2 Trial. The Lancet Oncology, 16, 908-918. http://dx.doi.org/10.1016/S1470-2045(15)00083-2

[11] Weber, J.S., D’Angelo, S.P., Minor, D., et al. (2015) Nivolumab versus Chemotherapy in Patients with Advanced Melanoma Who Progressed after Anti-CTLA-4 Treatment (CheckMate 037): A Randomised, Controlled, Open-Label, Phase 3 Trial. The Lancet Oncology, 16, 375-384. http://dx.doi.org/10.1016/S1470-2045(15)70076-8

[12] Felcht, M. and Thomas, M. (2015) Angiogenesis in Malignant Melanoma. Journal der Deutschen Dermatologischen Gesellschaft, 13, 125-136. http://dx.doi.org/10.1111/ddg.12580

[13] Chen, D.S. and Mellman, I. (2013) Oncology Meets Immunology: The Cancer-Immunity Cycle. Immunity, 39, 1-10. http://dx.doi.org/10.1016/j.immuni.2013.07.012

[14] Hodi, F.S., Lawrence, D., Lezcano, C., et al. (2014) Bevacizumab plus Ipilimumab in Patients with Metastatic Melanoma. Cancer Immunology Research, 2, 632-642. http://dx.doi.org/10.1158/2326-6066.CIR-14-0053

[15] Weide, B., Elsasser, M., Buttner, P., et al. (2012) Serum Markers Lactate Dehydrogenase and S100B Predict Independently Disease Outcome in Melanoma Patients with Distant Metastasis. British Journal of Cancer, 107, 422-428. http://dx.doi.org/10.1038/bjc.2012.306

[16] Omholt, K., Platz, A., Kanter, L., Ringborg, U. and Hansson, J. (2003) NRAS and BRAF Mutations Arise Early during Melanoma Pathogenesis and Are Preserved throughout Tumor Progression. Clinical Cancer Research, 9, 6483-6488.

[17] Lang, J. and MacKie, R.M. (2005) Prevalence of Exon 15 BRAF Mutations in Primary Me- 
lanoma of the Superficial Spreading, Nodular, Acral, and Lentigo Maligna Subtypes. Journal of Investigative Dermatology, 125, 575-579.

http://dx.doi.org/10.1111/j.0022-202X.2005.23833.x

[18] von Moos, R., Seifert, B., Simcock, M., et al. (2012) First-Line Temozolomide Combined with Bevacizumab in Metastatic Melanoma: A Multicentre Phase II Trial (SAKK 50/07). Annals of Oncology, 23, 531-536. http://dx.doi.org/10.1093/annonc/mdr126

[19] Middleton, M.R., Dalle, S., Claveau, J., et al. (2016) Real-World Treatment Practice in Patients with Advanced Melanoma in the Era before Ipilimumab: Results from the IMAGE study. Cancer Medicine, 5, 1436-1443. http://dx.doi.org/10.1002/cam4.717

[20] Minor, D.R. (2012) Bevacizumab Advanced Melanoma (BEAM) Was a Positive Trial. Journal of Clinical Oncology, 30, 2023-2024. http://dx.doi.org/10.1200/JCO.2012.41.7477

[21] Masunaga, S., Liu, Y., Tanaka, H., et al. (2011) Reducing Intratumour Acute Hypoxia through Bevacizumab Treatment, Referring to the Response of Quiescent Tumour Cells and Metastatic Potential. The British Journal of Radiology, 84, 1131-1138. http://dx.doi.org/10.1259/bjr/38457938

[22] Kerbel, R.S. (2008) Tumor Angiogenesis. The New England Journal of Medicine, 358, 20392049. http://dx.doi.org/10.1056/NEJMra0706596

[23] Corrie, P.G., Marshall, A., Dunn, J.A., et al. (2014) Adjuvant Bevacizumab in Patients with Melanoma at High Risk of Recurrence (AVAST-M): Preplanned Interim Results from a Multicentre, Open-Label, Randomised Controlled Phase 3 Study. The Lancet Oncology, 15, 620-630. http://dx.doi.org/10.1016/S1470-2045(14)70110-X

[24] Syrigos, K.N., Karapanagiotou, E., Boura, P., Manegold, C. and Harrington, K. (2011) Bevacizumab-Induced Hypertension: Pathogenesis and Management. BioDrugs, 25, 159-169. http://dx.doi.org/10.2165/11590180-000000000-00000

[25] Gonzalez-Cao, M., Viteri, S., Diaz-Lagares, A., et al. (2008) Preliminary Results of the Combination of Bevacizumab and Weekly Paclitaxel in Advanced Melanoma. Oncology, 74, 12-16. http://dx.doi.org/10.1159/000138351

[26] Perez, D.G., Suman, V.J., Fitch, T.R., et al. (2009) Phase 2 Trial of Carboplatin, Weekly Paclitaxel, and Biweekly Bevacizumab in Patients with Unresectable Stage IV Melanoma: A North Central Cancer Treatment Group Study, N047A. Cancer, 115, 119-127. http://dx.doi.org/10.1002/cncr.23987

[27] Kottschade, L.A., Suman, V.J., Perez, D.G., et al. (2013) A Randomized Phase 2 Study of Temozolomide and Bevacizumab or Nab-Paclitaxel, Carboplatin, and Bevacizumab in Patients with Unresectable Stage IV Melanoma: A North Central Cancer Treatment Group Study, N0775. Cancer, 119, 586-592. http://dx.doi.org/10.1002/cncr.27760

[28] Spitler, L.E., Boasberg, P., O’Day, S., et al. (2015) Phase II Study of Nab-Paclitaxel and Bevacizumab as First-Line Therapy for Patients with Unresectable Stage III and IV Melanoma. American Journal of Clinical Oncology, 38, 61-67. http://dx.doi.org/10.1097/COC.0b013e318287bbae

[29] Ferrucci, P.F., Minchella, I., Mosconi, M., et al. (2015) Dacarbazine in Combination with Bevacizumab for the Treatment of Unresectable/Metastatic Melanoma: A Phase II Study. Melanoma Research, 25, 239-245. http://dx.doi.org/10.1097/CMR.0000000000000146 
Submit or recommend next manuscript to SCIRP and we will provide best service for you:

Accepting pre-submission inquiries through Email, Facebook, LinkedIn, Twitter, etc. A wide selection of journals (inclusive of 9 subjects, more than 200 journals)

Providing 24-hour high-quality service

User-friendly online submission system

Fair and swift peer-review system

Efficient typesetting and proofreading procedure

Display of the result of downloads and visits, as well as the number of cited articles

Maximum dissemination of your research work

Submit your manuscript at: http://papersubmission.scirp.org/

Or contact jct@scirp.org 